\title{
Experiencias lúdicas \\ en la enseñanza de la economía - ludiconomía
}

\author{
Carlos Ariel Ramírez Triana ${ }^{25}$
}

\section{Introducción}

$\mathrm{H}$ ay varias maneras de mejorar los procesos de enseñanza-aprendizaje. No hay un manual ni recetario que proporcione una solución única frente a la problemática de impartir clases de economía que resulten amenas, innovadoras y, sobre todo, que garanticen el aprendizaje en un grupo de estudiantes.

En cada caso es necesario hacer una revisión del contexto que rodea el problema a tratar. Esto permite entender su naturaleza y a partir de allí puede surgir la respuesta de cómo abordarlo, y garantizar su solución. Este tipo de experiencias que se documentan a continuación son fruto de un ejercicio de indagación permanente que no se agota en el presente ejercicio de investigación. Se puede indicar que las estrategias presentadas en este capítulo han dado resultados positivos, pero son susceptibles de mejora con su aplicación sucesiva en el aula.

Este capítulo muestra una reflexión breve a manera de revisión de literatura, frente a la sintomatología más recurrente en aulas de economía en torno a su enseñanza o su práctica pedagógica, ampliamente centrada en la clase magistral y, a su vez, ofrece un conjunto de experiencias que se proponen para hacer frente a los inconvenientes que supone la ausencia de aplicación de estrategias didácticas para la enseñanza aprendizaje en las aulas de economía.

25. PhD en Management de la Universidad de Macquarie (Australia) MSc, BSc Economía (Universidad Nacional de Colombia). Profesor Investigador Titular de la Facultad de Negocios, Gestión y Sostenibilidad de la Institución Universitaria Politécnico Grancolombiano. 


\section{Revisión de literatura}

El ejercicio docente en la educación superior es un desafío que va más allá de su facilitador principal: el profesor. Para poder hacer una "buena clase", o desarrollar una "buena asignatura", se requiere una participación a nivel de equipo y coordinación de programa para poder identificar aquellos puntos clave (debilidades y fortalezas), con las que cuentan los estudiantes, es decir, potenciar el factor humano.

Estas particularidades pueden haber sido herencia de su formación básica o pueden ser también fruto de la formación universitaria a las que haya sido expuesto el estudiante. A propósito, como lo señala David Ausubel, citado por Ojeda, desde el constructivismo: "lo que se aprende depende en gran medida de lo que se conoce y, al aprender, relacionamos la nueva información con conceptos relevantes que existían previamente en nuestra estructura cognitiva" (Ojeda et al., 2007).

Así mismo es fundamental que el cuerpo docente, bajo un buen liderazgo, se dé a la tarea de actualizar constantemente contenidos programáticos y que estos cambios se reflejen en el desarrollo de nuevos materiales y aproximaciones a la enseñanza.

El puente que se extiende desde el aprendizaje (objetivo) y la enseñanza (medio), no siempre es claro. En este sentido, se indica que el aprendizaje va ligado a la percepción y el entendimiento del mundo (Marton y Booth, 2013). Pero trasciende de lo anterior a un escenario en donde se requiere el buen dominio de principios abstractos, entendimiento de demostraciones, la recordación de hechos puntuales, la adquisición de métodos, técnicas y aproximaciones, razonamiento, debate, entre otros (Fry, Ketteridge y Marshall, 2008). En definitiva, el camino entre el conocimiento que posee el docente y ser materializado en implicaciones prácticas de enseñanza, es complejo.

Ahora bien, el estudiantado es heterogéneo en sus expectativas y orígenes. Esto da como resultado una tarea especialmente compleja, ya que no todos aprenden de la misma manera o traen la misma disposición para capturar los materiales o metodologías preparados por los docentes.

Desde la academia, particularmente desde la psicología, se ha tratado de establecer qué tan diferentes son los procesos de aprendizaje de los adultos, en comparación con los procesos de los niños, que están ampliamente explorados y documentados. La razón para tal pregunta descansa sobre el supuesto de que el carácter de los adultos difiere ampliamente del de los niños, y es así que surge toda una corriente de esfuerzo acerca de los procesos de enseñanza-aprendizaje para personas adultas: la andragogía. Este término, acuñado por Malcom Knowledges, y que surge como contraposición a la pedagogía, se define como el arte y ciencia de ayudar a los adultos a aprender (Merriam, 2001). Sin embargo, en este capítulo no se hará énfasis en estas disertaciones filosóficas acerca de la terminología, sino que se expondrán casos aplicados en el aula, en torno a temas enseñados en economía. 
Para ello, se identifican varias problemáticas latentes, que no son ajenas a otras disciplinas, pero que han sido el motor de estas propuestas en aulas de economía, descritas en el presente capítulo. En particular, se hace referencia al plagio, a métodos de estudio (particularmente el uso - o abuso de la memoria), y usos de lenguaje, la baja motivación para atender a las clases y/o realizar trabajos.

Respecto del plagio, la literatura académica es bastante amplia. Un documento muy completo al respecto es el trabajo de Sureda, Comas y Morey (2009), en donde se indica que hay varias causas iniciales documentadas que dan cuenta del por qué los alumnos incurren en prácticas de copia: necesidad hacia la nota, pereza y desorganización frente a la redacción de trabajos, facilidad y eficiencia al encontrar el trabajo directamente en Internet, y finalmente, por desconocimiento de las reglas y normas de citación.

Sin embargo, en un estudio más exhaustivo, expuesto por los autores, también se presentan otras raíces en torno al mismo asunto: interiorización de falsos valores, lo que el estudiante puede presentar como "viveza"; desmotivación, al considerar que el requisito no es importante; desafío, intentando mirar qué sucede si no se realiza esta parte de la tarea; desconocimiento, no tanto frente a la regla sino a su implicación moral, es decir, que no ven lo malo de esta práctica; y finalmente, en el análisis riesgo-beneficio el estudiante usualmente ve que a pesar de reconocer que es una mala práctica, considera que la posibilidad e impacto del castigo son menores, frente al éxito obtenido de no ser detectado.

Otro de los dinamizadores de este capítulo está en el uso de la memoria como técnica de estudio. El uso de la memoria ha sido supremamente bien valorado como indicador de aprendizaje dentro de las mediciones de la efectividad de la educación en modelos antiguos (de hace dos o más décadas). En algunos sitios la repetición y el aprendizaje memorístico continúan siendo ampliamente sopesados por encima del entendimiento, procesamiento de datos y solución de problemas. Por ejemplo, en la educación en el mundo oriental recientemente se busca dirigir al estudiante a la resolución de exámenes y al uso de la memoria para lograr estos resultados. Esto genera una práctica en donde el docente debe apresurarse en el recorrido del libro de texto, y se descuidan procesos de entendimiento, dadas las restricciones de tiempo (Hassan, Jamaludin, Sulaiman y Baki, 2010). Estos mismos objetivos muchas veces vienen siendo aplicados en el sistema educativo colombiano, particularmente en la educación secundaria, de tal suerte que cuando se solicitan procesos cognitivos de mayor complejidad, en alumnos universitarios, se da una interrupción, ya que las habilidades relacionales y de inferencia no han sido lo suficientemente trabajadas por dar cabida a la repetición.

Lo anterior es grave en asignaturas que están fuertemente soportadas en el lenguaje matemático, ya que difícilmente se podría "aprender" una demostración, como quien recita una poesía. Para entender la argumentación a partir de ecuaciones se requiere un proceso de lectura acompañada de ejercicio simultáneo (es decir, realizar el camino lógico 
sugerido por el autor del texto que se esté trabajando). Se necesita completar los baches (gráficos y de ecuaciones), que muchas veces en los textos se presentan como conclusiones obvias; hacer cambios de lenguaje, para facilitar la comprensión. En ningún caso, para el estudio de este tipo de asignaturas, basta con "pasar los ojos" por el escrito, para lograr su comprensión.

Los académicos han referenciado que tanto las grandes inversiones educativas (como la construcción de una biblioteca atractiva), así como los cambios pequeños en las prácticas pedagógicas (el uso de una caricatura), pueden tener efectos profundos en la motivación de los estudiantes (Anderman \& Dawson, 2011). Esta motivación puede tener una naturaleza intrínseca o extrínseca, lo cual brinda una arista más compleja a la hora del diseño de una estrategia para llamar la atención o activar ese gatillo que dé como resultado una mejor disposición al aprendizaje. La motivación puede ser el vehículo de muchos propósitos en el aula: realización de tareas de una tipología particular, alcance completo de objetivos, manejo de expectativas(Anderman y Dawson, 2011), de suerte que no existe unicidad en el camino a plantear en este frente. Se debe ser cuidadoso frente al momento en que se lance una propuesta, el background y homogeneidad académica con la que cuenta el grupo, entre otros factores. Un reto, un caso, una pieza musical pueden ser los detonantes de estos procesos.

Dado todo lo anterior, a continuación se presentan algunas iniciativas que se han desarrollado en cursos de economía en la Institución Universitaria Politécnico Grancolombiano (de distintos niveles de profundidad y de avance en la malla curricular), y el recuento se hace siguiendo básicamente la cronología bajo la cual se han implementado.

\section{Aplicaciones en el aula}

\section{Experiencia 1}

Nombre de la experiencia: Cuentos microeconómicos.

Contexto: la elaboración de trabajos académicos para finalización de semestre generalmente se caracteriza por la poca apropiación de conceptos por parte de los estudiantes y por la tendencia al uso del plagio para poder cumplir con el objetivo de entregar. La idea de esta experiencia, ejecutada en el año 2004 para el curso de microeconomía básica, con aproximadamente 20 estudiantes, fue lograr un trabajo final que superara las dos problemáticas señaladas. 
Cómo se planteó la solución: en esta primera aproximación se buscó evaluar el trabajo individual, generando un acercamiento contextual a los conceptos y, en segundo lugar, se diseñó un mecanismo para enfrentar el asunto del plagio.

Antes de proceder con la solución se debió dar respuesta a dos preguntas básicas:

1. ¿Por qué es complicado entender el manejo conceptual propio de la microeconomía básica?

2. ¿Por qué resulta el plagio como el camino más recurrente por los estudiantes para hacer frente a la presentación de trabajos escritos?

La respuesta a la primera pregunta descansa sobre el hecho de que muchas veces se realiza una enseñanza abstracta de la microeconomía. Esto quiere decir que los estudiantes no logran establecer la conexión entre los conceptos y la realidad que los rodea. Adicionalmente, el uso de la memoria ha sido uno de los mecanismos típicos de estudio en el contexto colombiano, sin embargo, proporciona resultados fragmentados y en muchos casos descontextualizados. La memoria está sobrevalorada y malentendida por algunas personas dentro de la academia, pero también es, en ocasiones, subestimada. Se requiere el uso de la memoria moderado y con un enfoque de aprendizaje significativo y funcional (Coll, 1992).

Respecto del segundo interrogante se puede referir que el manejo de fuentes bibliográficas y de los procesos de citación usualmente se presenta al estudiante como una solicitud o un requerimiento, pero con frecuencia no se le explica la utilidad de este proceso. Del mismo modo, los trabajos tradicionales abundan en contextos digitales, de tal suerte que si se le pide al estudiante algo que ya está hecho, en donde el valor agregado individual no es sustancial, le resulta atractivo hacer uso del plagio.

Esto se corrobora en el argumento expuesto por Sureda et al. (2009):

(...) los profesores universitarios creen que los alumnos plagian porque con Internet es muy fácil hacerlo; los estudiantes no valoran el esfuerzo y el trabajo; no gestionan adecuadamente el tiempo; no saben cómo realizar trabajos académicos; y además los profesores no hacen un seguimiento adecuado de estos trabajos. (Citado en Egaña, 2012, p.2)

Inclusive, no es de práctica común que los docentes integren bibliografía, más allá de los manuales en la presentación del sílabo de la asignatura. Esto hace que las estrategias de búsqueda bibliográfica sean limitadas y que no exista suficiente familiaridad sobre cómo hacer procesos de referenciación de manera adecuada.

$\mathrm{Al}$ estudiante se le debe explicar que la referenciación y correcta citación es un proceso importante. No solamente lo es por el reconocimiento al trabajo propio y ajeno; en ese sentido hay una amplia discusión en la literatura: el plagio va más allá del individuo y afecta también a la institución que lo permite, y su práctica desemboca en productos de baja originalidad y calidad, en general. Es responsabilidad de las universidades hacer 
la reflexión al respecto y formar no solo en lo académico, sino en lo ético y moral de este asunto (Batane, 2010).

No se pretende, mediante el argumento anterior, justificar este comportamiento no ético, pero sí, por lo menos, entender el actuar del alumno frente a las exigencias que se le hacen en el aula de clase y a los insumos con los que cuenta para atenderlas.

Metodología de la propuesta: en este trabajo se ofreció al estudiante un listado de aproximadamente 70 términos o expresiones que fueron desarrollados en la asignatura Microeconomía básica. Dentro de ellos se puede encontrar elementos sencillos y de manejo cotidiano como oferta, demanda, equilibrio, monopolio, etc.; pero también se ubicó dentro de la lista terminología puramente técnica como curvas de indiferencia, isocuantas, tasa marginal de sustitución, equimarginalidad, entre otros.

El estudiante debió usar este listado como insumo para redactar un cuento a la manera tradicional, en donde se desarrolle una historia con introducción, nudo y desenlace.

Las instrucciones dadas implicaban que se debía hacer uso de al menos 50 términos así:

- Uso dentro del contexto microeconómico: al menos 30 términos, resaltados con un color particular (por ejemplo, rojo).

- Uso libre, no necesariamente microeconómico: al menos 20 términos, resaltados con un color particular (por ejemplo, azul).

Lo anterior quiere decir que si el estudiante quiso hacer uso de la palabra oferta dentro de su cuento, pudo hacerlo bajo el contexto propio microeconómico en el primer uso, por ejemplo:

"La princesa Helena se encontraba enormemente decepcionada al enterarse que la oferta

de los tulipanes morados, que tanto le gustaban, venía en franco declive año tras año, desde la coronación de la malvada bruja glaciar."

O pudo hacer un uso no microeconómico, si no tenía claridad de qué hacer con ella o cómo usarla dentro del cuento, por ejemplo:

"Y se venía presentando una rivalidad milenaria entre los fieros habitantes de la aldea oferta, con aquellos calmos personajes que moraban en las colinas de aquella región."

Un elemento final que fue incorporado dentro de esta práctica fue la elaboración de un glosario a través del parafraseo. Esto quiere decir que los estudiantes, dentro de su propuesta, debieron hacer la búsqueda documental de los diferentes términos seleccionados en piezas de literatura técnica, debidamente referenciada, y adicional a ello debieron hacer una interpretación del texto escogido, para que, en sus palabras, compartieran al lector final, su interpretación. 
Resultados: los resultados que se dieron fueron variados: por una parte, se lograron algunos escritos que básicamente fueron desarrollados bajo el esqueleto de un trabajo previo (cuento, película, libro), pero se incorporó el efecto requerido del uso de las palabras como se propuso inicialmente. Así pues, se lograron piezas tipo Caperucita o tipo Matrix, en donde el estudiante exponía y apropiaba el lenguaje y conceptos teóricos de la microeconomía.

Una segunda corriente presentó un componente creativo mucho más alto. Allí los estudiantes se comprometieron mucho más con la tarea de imaginar la historia. Se pudo evidenciar falencias en estilos de escritura, redacción y ortografía, sin embargo, el componente conceptual fue abordado con propiedad. A pesar de que no todas las piezas resultaron cautivadoras, se ponderó fuertemente el aspecto creativo a la hora de la calificación.

Finalmente, en ninguno de los casos se pudo evidenciar presencia de plagio, que era uno de los objetivos iniciales, y así mismo en este grupo se pudo ver resultados muy positivos, respecto de otros grupos, en un instrumento de evaluación de preconceptos de microeconomía que se realizó a inicios del semestre inmediatamente siguiente.

\section{Experiencia 2}

Nombre de la experiencia: Juegos microeconómicos.

Contexto: siguiendo con la dinámica de los cuentos se buscó otro mecanismo mediante el cual los estudiantes pudieran hacer aproximación a la apropiación conceptual de la teoría microeconómica, sin incurrir en trabajos bajo esquemas tradicionales y procurando evitar al máximo la posibilidad de plagio en la elaboración de estos. Adicionalmente se buscó que se propiciara un ambiente motivacional positivo para la elaboración de la tarea.

En esta oportunidad se encontró como un valor agregado la posibilidad de trabajar en grupos de máximo cuatro estudiantes, ya que facilitaría tanto la elaboración de los "productos" o juegos, como la implementación de los mismos.

Esta práctica se hizo durante cuatro semestres con dos aproximaciones diferentes. Los grupos que fueron sujeto de esta experiencia variaban de 25 a 40 estudiantes.

¿Cómo se planteó la solución?: el uso de una aproximación distinta, no replicable de Internet, llevó a la evasión del problema del plagio, aunque se conservó la revisión documental y correcta referenciación. El componente motivacional y de afianzamiento conceptual fue dado por medio de la lúdica. Esta experiencia no es vanguardista ni aislada en este sentido. Algunos ejemplos exitosos han sido documentados frente a la enseñanza de conocimientos técnicos mediante la lúdica (por ejemplo, Jiménez, Dinello y Alvarado, 2004; Palacino Rodríguez, 2007; Ramirezparis Colmenares, 2009). Desde el Ministerio de Educación Nacional se resalta el rol positivo del juego en los ambientes de aprendizaje: 
Los juegos inspiran a los estudiantes a pensar, a crear y recrear con actividades que contribuyen al desarrollo de la atención y la escucha activa, el seguimiento de instrucciones y el compromiso para cumplir reglas, para, de esta manera, comprender en la vivencia y convivencia, en la acción y corrección. (Cepeda Ramírez, 2017)

Metodología: se hizo una revisión conceptual y de aplicación práctica de la temática de la microeconomía básica por medio de juegos en dos aproximaciones diferentes.

En la primera aproximación, durante el año uno se les solicitó a los estudiantes que diseñaran una propuesta o prototipo de juego de tablero que promoviera, durante el desarrollo de este, el estudio o aplicación de conceptos relacionados con la asignatura. Una vez la propuesta de los diferentes grupos fuera revisada y avalada por el docente, se procedía con la puesta en marcha de ella: es decir, hacer o construir el prototipo de juego o tablero, con sus respectivas fichas, cartas, y/o demás elementos propios del juego.

Un elemento primordial durante el desarrollo de esta propuesta estuvo en la elaboración del manual de instrucciones, que no solo debía consignar el reglamento intrínseco para la implementación del juego propuesto (en donde se debía plantear el objetivo primordial de juego, la manera de alcanzarlo, el establecimiento de turnos, la asignación de puntos, etc.), sino que dentro de este "manual" se debía incluir un compendio teórico microeconómico suficiente para que cualquier jugador, mediante un breve repaso, pudiera participar en las preguntas que se iban desarrollando a lo largo del juego.

En la segunda aproximación, en el año 2, se hace una ligera variante en donde el diseño o puesta en marcha debía contemplar propuesta o prototipos de juegos de campo. En esta dinámica se buscó romper la dinámica de alcance del espacio físico del aula. El proceso de revisión de este tipo de juegos supuso un reto adicional, dado que los espacios por fuera del aula entre semana estaban ocupados simultáneamente para la realización de otras alternativas culturales o deportivas.

Resultados: en términos comparativos se cubre la totalidad de un trabajo tradicional, si se tiene en cuenta que este trabajo involucra, implícitamente, una justificación, un desarrollo de objetivos, la metodología es impuesta y guiada por el docente, mas no la obtención de producto final, resultados y conclusiones. El producto final, en términos de presentación, es diverso y con diferentes niveles de profundización y énfasis. Sin embargo, cierta homogenización se hizo presente: en la mayoría de los productos o juegos, independiente de la aproximación (mesa o campo), se dio la incorporación de pregun- 
tas de selección múltiple, como elemento indispensable de avance a través del juego. En ocasiones aisladas, el jugador (o estudiante) era retado a la elaboración, ilustración o argumentación como requisito para tomar parte del desarrollo del juego.

En el caso de los juegos de campo, fue tanta la motivación de los estudiantes que, bajo propuesta de ellos, se utilizó tiempo fuera de la universidad (domingo en la mañana), para poder evaluar los diferentes juegos.

Esquirla pedagógica: evaluación y coevaluación: en estas propuestas anteriores, dada la dinámica de grupos, se buscó la forma en que todos los estudiantes participaran en los trabajos que habían sido elaborados por sus pares. Los juegos fueron probados y evaluados dentro del grupo, por parte del profesor y por algunos de sus compañeros jugadores. Este elemento no fue sorpresivo, sino que fue anunciado con antelación en aras de forzar a los distintos grupos a tener una responsabilidad de entrega no solamente hacia el docente, sino con su grupo de pares.

La coevaluación se diseñó en términos positivos y propositivos, tanto de fondo como de forma. En el formato de evaluación diseñado se invitaba a los "evaluadores" a cuantificar el esfuerzo de sus pares en términos de presentación, creatividad alrededor del juego y claridad y profundidad de las preguntas. Sin embargo, dicha valoración cuantitativa debía ser justificada de forma argumentada, en donde el "evaluador" de forma anónima debía indicar a los creadores del juego cómo mejorar el mismo, a partir de sus recomendaciones. A pesar de los vínculos de amistad entre los miembros del curso, no se dieron prerrogativas entre ellos a la hora de evaluar. La coevaluación, así como otras prácticas que vinculan al estudiante en los procesos de evaluación, potencian el aprendizaje y dinamizan los procesos educativos generados, nutren el análisis crítico y la autocrítica, genera autonomía del alumnado, traducida en la formación de personas responsables y el desarrollo de una educación democrática y, finalmente, acentúa las convicciones educativas y afianza los proyectos curriculares (Pastor, Pascual y Martín, 2005).

\section{Experiencia 3}

Nombre de la experiencia: Sancocho intercambiable

Contexto: el modelo de intercambio puro, presentado bajo la Teoría de equilibrio general es de las representaciones más complejas del desarrollo microeconómico a las que un estudiante de pregrado en economía se ve enfrentado. En él se trabaja sobre las bases de maximización de Marshall, con el objetivo de lograr la máxima utilidad para dos agentes, con la diferencia que allí tanto el precio como el presupuesto no son dados de forma explícita exógenamente, 
sino que son propuestos por medio de un conjunto de activos con los que cuentan los agentes y que se denominan dotaciones iniciales. Con la información anterior se busca que el estudiante halle las cantidades óptimas de los bienes en cuestión (en términos de los precios, es decir, las demandas Marshallianas), y que se vacíen los mercados (Ley de Walras), para poder hallar finalmente un vector de precios, que permitirá (al ser remplazado en las demandas), dar numéricamente con el equilibrio de Walras (Lozano, Villa y Monsalve, 1999).

El uso más frecuente de este ejercicio se da bajo condiciones artificiales, de funciones de utilidad tipo Cobb-Douglas, que se comportan matemáticamente bien y garantizan la existencia y unicidad del equilibrio.

El modelo como tal ha generado una serie de críticas, no tanto en torno a la lógica de su desarrollo, pero sí en torno a sus fundamentos. Por ejemplo, se ataca el extremo de la racionalidad de los agentes, la perfección de la información, entre otros (Rueda, 2009). Por su parte, Cataño se centra en otras fallas: en particular resalta la falta de replicabilidad del modelo en la realidad y la inexistencia de pruebas de convergencia hacia el equilibrio (Cataño, 1997, 2004).

A pesar de lo anterior, la bibliografía acerca del tema es escasa, y son pocos los esfuerzos editoriales que de manera aislada dedican un libro de texto completo a tratar la Teoría del equilibrio general; a lo sumo, este tema es un capítulo dentro de un texto de microeconomía intermedia.

En el caso del Politécnico Grancolombiano se ha hecho uso del material de Monsalve para la enseñanza de esta asignatura (Monsalve, 1999), y no ha habido cabida para una adecuación anterior en términos de uso de lenguaje matemático (por ejemplo, el uso de Varian para microeconomías anteriores). De hecho, se ha usado para ello materiales desarrollados internamente y libros de texto que manejan niveles básicos de profundidad. En el libro de texto de Monsalve el nivel de abstracción usado es elevado y genera frecuentemente aversión y confusión en el estudiante a la hora de aproximarse a su lectura. No obstante, se reconoce que es una obra muy completa, bien elaborada y con conclusiones importantes. Genera un desafío interesante para estudiantes y docentes, tanto en términos del desarrollo algebraico como en la línea argumental de lo que expone. Adicionalmente, se considera que proporciona muy buenas bases para ejercicios de modelamiento en economía, tanto a nivel microeconómico como macroeconómico.

Los cursos en los que se ha planteado esta metodología se componen de 8 a 12 estudiantes. Se ha aplicado durante varios semestres no consecutivos entre los años 2016 a la fecha. En estos cursos se ha visto cómo los estudiantes, frente al escaso entendimiento de la asignatura, buscan refugio en la memorización para garantizar la aprobación de la misma (lo cual es un camino equivocado, según la evidencia empírica).

Lo ideal es trabajar de forma individual, con interacción del grupo (es decir, cada estudiante requiere de sus propios materiales, pero debe construir colectivamente la respuesta 
mediante sus aportes personales). En grupos un poco más grandes se sugeriría hacer subdivisiones del grupo (no más de 8 personas), para realizar la actividad.

¿Cómo se planteó la solución?: existe un riesgo fuerte en este tipo de asignaturas, en donde el estudiante se apropie de la mecánica del ejercicio y al final termine ejecutando una rutina de solución y no llegue al análisis y solución del problema. Introducir cambios ligeros en el planteamiento de este tipo de ejercicios lleva usualmente al estancamiento, simplemente porque no se entiende la naturaleza del mismo.

Por lo anterior se hace un ejercicio lúdico que permite acercarse desde un ángulo diferente, y que es más próximo a la cotidianidad del estudiante. El cambio de lenguaje acerca mucho más al estudiante al entendimiento del tema en cuestión.

Metodología: cada estudiante recibe un paquete de parte del profesor. Este paquete consta de dos elementos básicos: a. Una carta imaginaria y personal escrita por una tía alcahueta, indicando la receta de la sopa favorita del estudiante; en ella se da una relación exacta de los ingredientes que se requieren para poder lograr una porción de la misma; b. Un conjunto de algunos gráficos impresos que representan los ingredientes requeridos para su elaboración (ej: pollo, papas, cilantro, aguacate, carne, yuca, etc.). A cada estudiante se le entrega una receta diferente y un grupo de ingredientes incompleto, lo cual le imposibilita hacer la receta autárquicamente. No es necesario que la variedad de ingredientes iguale al número de estudiantes participantes. Se puede ambientar la actividad indicando al estudiante que su origen es campesino y los productos que tiene los lleva a la plaza de mercado. También se debe presuponer la no existencia de dinero. Simultáneo a lo anterior se puede poner música folclórica o popular, que relaja bastante el ejercicio.

El objetivo del ejercicio es procurar hacer la mayor cantidad de recetas (es decir, conseguir n veces el número de ingredientes completos), por parte de cada participante, mediante el intercambio. El ejercicio cuenta con dos fases: en la primera se prohíbe la divulgación de las recetas, y en la segunda ya se puede conocer esta información.

Se invita al estudiante a hacer el paralelo de identificación de cada uno de los elementos de la actividad con lo presentado en el modelo formal (utilidad, demandas, precios relativos, dotaciones iniciales, etc.). Igualmente se busca, sobre todo en la segunda fase del ejercicio, un acercamiento a la eficiencia (al conocer colectivamente las recetas), buscando el intercambio más cercano al cumplimiento de la Ley de Walras y asignaciones paretianas. El ideal de la actividad (que no siempre se logra), es que cada estudiante logre realizar la valoración de su canasta inicial por medio del reconocimiento y cuantificación de los términos de intercambio (es decir, la obtención de los precios relativos), y finalmente la valoración de su utilidad al final de ejercicio. 
Resultados: el componente argumentativo que rodea la formalidad del modelo incrementa sustancialmente, en el estudiante, después del planteamiento de la actividad. Del mismo modo, el estudiante se siente un poco más empoderado desde su nuevo entendimiento del modelo para elevar cuestionamientos acerca de sus supuestos básicos, objetivos y alcances. El ejercicio relaja la aproximación algebraica y se encuentra, sorprendentemente, mayor fluidez en el desarrollo de los problemas.

\section{Experiencia 4}

Nombre de la experiencia: Elecciones económicas.

Contexto: la enseñanza de las corrientes de pensamiento económico en cursos introductorios o de fundamentos de economía, usualmente se presta para la división de diferentes temáticas entre grupos diversos de estudiantes. A pesar de lo anterior, con frecuencia los niveles de atención entre pares, sobre todo entre los primeros semestres, son reducidos.

El presente ejercicio se dio a la luz de las recientes elecciones presidenciales (2018), y se hizo uso de todo el impulso mediático, programático y de campaña que rodeó al curso de Introducción a la economía, de aproximadamente 40 estudiantes.

¿Cómo se planteó la solución?: aprovechando la coyuntura electoral y el interés que la misma generó en los estudiantes, se planteó un ejercicio en el cual, por grupos, se expusieron las diferentes corrientes de pensamiento económico, como si ellas fuesen un partido político. La culminación del ejercicio se dio con las votaciones que se llevaron a cabo dentro del curso.

Hacer uso de una propuesta de presentación diferente y bajo la introducción del sentido competitivo alrededor del trabajo se logró capturar la atención de los estudiantes, incrementar sus niveles de compromiso y crítica y el compromiso con una corriente filosófica completamente argumentada. El trasfondo pedagógico que respaldó esta iniciativa fue dado mediante la motivación intrínseca de los estudiantes, en razón del momento político y de la importancia nacional de dichas elecciones. Este tipo de motivaciones debe ser aprovechado en el momento adecuado (Anderman y Dawson, 2011).

Metodología: el grupo fue inicialmente dividido en subgrupos, y a cada subgrupo le fue asignada una corriente de pensamiento económico para investigar y presentar, así:

Cada subgrupo debió elaborar con la información recabada un perfil de un candidato, indicando quién es el personaje y cuáles son sus ideas principales. De la misma manera, debió darse una identificación y presentación de los asociados políticos, un eslogan y una 
infografía. Este último elemento fue socializado en clase, a manera de campaña, y cargado en la página de Moodle (para efectos de estudio colectivo posterior y preparación para el examen respectivo). En este mismo sentido se elaboró un folleto y se dio un espacio creativo para presentar al "electorado" (es decir, al resto de subgrupos). Este espacio creativo consistió en crear una canción, fan-page, entrevista, comercial, en donde se realce el espíritu del partido asignado.

Al curso en total se le dio un cronograma completo, en donde se indicó cada uno de los hitos importantes del ejercicio (presentación de la propuesta, conformación de grupos, asignación de temas, socialización ante los electores y, finalmente, votación).

La votación se dio por medio de un tarjetón que se le entregó a cada estudiante del grupo, en donde podía plasmar dos votos para tener validez (presumiendo que votaran al menos una vez por su grupo y viéndose obligados a votar por otro grupo). Se armó puesto de votación, se asignaron jurados y se realizó conteo público en una aplicación de Excel diseñada por el docente.

Resultados: en términos pedagógicos se pudo ver a un grupo comprometido y motivado con la tarea. Se realizaron campañas interesantes y se pudo transmitir de forma más amena lo que en otros semestres costaba bastante. El examen reflejó académicamente una buena catalogación y discernimiento de la información presentada, y se vio reflejado en el promedio general del examen cómo el refuerzo de diferentes presentaciones fijó las ideas (sobre todo del espacio creativo), tanto entre quienes hicieron las diferentes presentaciones como entre quienes estuvieron presentes en las mismas.

\section{Experiencia 5}

Nombre de la experiencia: $\mathrm{Al}$ son que me toquen aprendo.

En esta última experiencia no se puede hacer un desarrollo puntual y estructurado como en las anteriores, dado que en diversos momentos y para diferentes temáticas funciona muy bien llamar la atención auditiva del estudiantado.

La música, en las clases de economía, se ha usado como elemento de relajación ante momentos de tensión (presentación de exámenes). Allí la música funge como motivación extrínseca. También se usa para la generación de un ambiente de trabajo distendido (por ejemplo, elaboración de talleres). En este caso, se propicia un ejercicio de tolerancia y respeto, ya que se solicita a los estudiantes que, en un pedazo de papel, de forma anónima, indiquen un par de canciones de su preferencia. Se escuchan todas, independientemente del género. Allí hay bastante motivación intrínseca hacia el trabajo. 
Finalmente, hay piezas aisladas que ilustran temas de clase y que permiten generar recordación y extracción de conclusiones por parte de los estudiantes. Por ejemplo, en microeconomía intermedia se presenta la temática de "La oferta de trabajo y la demanda de ocio". Habría varias canciones que pueden generar debate al respecto. En clase se ha usado "No hago más ná", del Gran Combo de Puerto Rico. Así como esta, hay muchas posibilidades de amenizar la clase y ofrecerla con un ambiente muy amable para la enseñanza y el aprendizaje. Este tipo de prácticas no deben ser consideradas como una involución de la formalidad, sino como un complemento y facilitador de la tarea docente.

\section{Conclusiones y reflexiones finales}

Estar frente a un grupo de estudiantes y dar una buena clase requiere coraje, dedicación, estudio y mucha disciplina. Sin embargo, pensar fuera de molde puede resultar en experiencias muy positivas, tanto para el estudiante en su desarrollo académico y cognoscitivo como para el docente en términos de enriquecimiento de sus prácticas pedagógicas.

Se debe explorar virtudes que pueden proporcionar ambientes distendidos, por medio del juego, la música y otros elementos que "pueden" salir de la cotidianidad de la clase estándar de economía, pero que se acerca más al día a día del estudiante. La extensión de este tipo de puentes debería facilitar los procesos de transmisión de conocimiento y la generación de nuevos procesos de razonamiento.

En términos de investigación en educación, las experiencias aquí presentadas son una muestra interesante de lo que se puede lograr en el aula de clase. Sin embargo, hace falta un poco más de seguimiento y cuantificación de resultados para proceder a hacer un análisis más profundo del impacto de estas prácticas. El establecimiento de un grupo control, para hacer comparativos, nutriría enormemente la discusión alrededor de los resultados acá presentados. Se deja esto como un propósito para futuras investigaciones sobre la enseñanza de la economía.

\section{Referencias}

Anderman, E. M., y Dawson, H. (2011). Learning with motivation. Handbook of Research on Learning and Instruction, 219214.

Batane, T. (2010). Turning to Turnitin to Fight Plagiarism among University Students. Journal of Educational Technology Asn Society, 13(2), 6-12. 
Cataño, J. F. (1997). El modelo de equilibrio general: ¿estático o estéril? Cuadernos de Economía, 16(27), 113-139.

Cataño, J. F. (2004). La teoría neoclásica del equilibrio general. Apuntes críticos. Cuadernos de Economía, 23(40), 175-204.

Cepeda Ramírez, M. R. (2017). El juego como estrategia lúdica de aprendizaje. Recuperado de: https://www.magisterio.com.co/articulo/el-juego-como-estrategialudica-de-aprendizaje

Coll, C. (1992). Psicología y currículum. Una aproximación psicopedagógica a la elaboración del currículum. México: Paidós. Recuperado de: http://rlillo.educsalud.cl/ Capac_Docente_Basico/psico curr1.doc

Egaña, T. (2012). Uso de bibliografía y plagio académico entre los estudiantes universitarios. Revista de Universidad y Sociedad del Conocimiento, 9, 18-30.

Fry, H., Ketteridge, S., y Marshall, S. (2008). A handbook for teaching and learning in higher education: Enhancing academic practice. New York: Routledge.

Hassan, A., Jamaludin, N., Sulaiman, T., y Baki, R. (2010). Western and Eastern educational philosophies. In 40th Philosophy of Education Society of Australasia conference, Murdoch University, Western Australia.

Jiménez, C., Dinello, R., y Alvarado, L. (2004). Recreación, lúdica y juego. Colección Aula Alegre. Bogotá: Editorial Magisterio.

Lozano, F., Villa, E., y Monsalve, S. (1999). Competencia perfecta: equilibrio walrasiano y óptimo de Pareto. En S. Monsalve (Ed.), Introducción a los conceptos de equilibrio en Economía. Bogotá: Universidad Nacional de Colombia.

Marton, F., y Booth, S. (2013). Learning and Awareness. New York: Routledge.

Merriam, S. B. (2001). Andragogy and self-directed learning: Pillars of adult learning theory. New Directions for Adult and Continuing Education, 2001(89), 3-14.

Monsalve, S. (1999). Introducción a los conceptos de equilibrio en economía. Bogotá: Universidad Nacional de Colombia.

Ojeda Cabrera, A., Cuéllar, D., Fe, E., González Landrián, L., Pinedo Melis, P., y Hernández Gener, M. E. (2007). Los mapas conceptuales: una poderosa herramienta para el aprendizaje significativo. Revista Cubana de Información En Ciencias de La Salud - Acimed, 15(5). Recuperado de: http://scielo.sld.cu/scielo.php?script=sci_ arttext\&pid=S1024-94352007000500009\&lng=es\&tlng=es.

Pastor, V. M. L., Pascual, M. G., \& Martín, J. J. B. (2005). La participación del alumnado en la evaluación: la autoevaluación, la coevaluación y la evaluación compartida. Rev. Tándem Didáctica Educ. Física, 17, 21-37. 
Ramirezparis Colmenares, X. (2009). La lúdica en el aprendizaje de las matemáticas. Zona Próxima - Revista del Instituto de Estudios en Educación Universidad Del Norte, (10).

Rueda, H. (2009). Debilidades de la teoría del equilibrio general. Revista Escuela de Administración de Negocios, 65(65), 197-121. Recuperado de: http://www.redalyc.org/ pdf/206/20612980006.pdf

Sureda, J., Comas, R., y Morey, M. (2009). Las causas del plagio académico entre el alumnado universitario según el profesorado. Revista Iberoamericana de Educación, 50, 197-220. 